between patients and proxies has been known in the West and this study investigated how perspectives differ in an Asian context.

Methods Twenty ACP discussion sheets completed with the patients, with or without a proxy decision maker and 20 ACP discussion sheets completed only with proxy, without the patient, were chosen at random for analysis. Thematic analysis was used to identify salient themes from both sets of discussion sheets and compared to understand the differences between decisions made by patients and proxies.

Results While overarching ideas on suffering and living well remain largely similar between the groups, there were marked differences in the area of medical interventions, place of care and religion. More proxies wanted comfort measures only for their loved ones, while patients tended to opt for additional interventions, such as intravenous medications or antibiotics. Similarly, while proxies preferred a trial of treatment at home or in a hospice before transferring to hospital, more patients wanted to be transferred to hospital immediately upon illness onset. Many patients mentioned religious beliefs as of importance whereas this was less mentioned by proxies.

Conclusions There are differences between the perspectives of patients and proxy decision makers in making ACPs. Future investigation should look at ways to align the proxy's perspectives with that of the patient especially in dyad pairs including means to reduce differences during ACP facilitation.

\section{P38 INTEGRATIVE LITERATURE-REVIEW ON SHARED DECISION-MAKING, ADVANCE CARE PLANNING, INCLUDING VALUES AND PREFERENCES OF ELDERLY PATIENTS WITH SYMPTOMATIC AORTIC VALVE STENOSIS}

NM Rogger*, D Drewniak, T Krones. University of Zürich, Zürich, Switzerland

10.1136/spcare-2019-ACPICONGRESSABS. 122

Background Only very few studies have been published on Shared Decision-Making (SDM) and Advance Care Planning (ACP) including high risk patients with aortic valve stenosis addressing transcatheter aortic valve replacement (TAVR), surgical valve replacement (SAVR) and palliative care as treatment options. Therefore, the aim of this study is to strengthen the basis for further investigations on this increasingly important theme.

Methods We currently perform a computerized integrated literature review of MedLine (PubMed), EMBASE and Cochrane databases, including among others the MeSH terms aged, frail, aortic valve stenosis, advance care planning, and sensitive search filters on health outcomes such as patient values and health care related quality of life. Qualitative and quantitative studies are both included. For quality evaluation we will use the method of systematic review developed by Hawker et al. (2002) and based on the Critical Appraisal Program (CASP) tool for qualitative research. Since a great variability of the study types can be expected, they will be systemized using thematic analysis approach.

Results We will present the ethical and practical relevance of this specific topic with regard to Advance Care Planning and Shared Decision-Making and present first results of our integrated literature review.

\section{P39 COLLUSION, ADVANCE CARE PLANNING AND THERAPEUTIC PRIVILEGE - PATERNALISM VIA THE BACK DOOR?}

${ }^{1} S$ Menon*, ${ }^{2}$ J Van Delden, ${ }^{3} \mathrm{~A}$ Campbell. 'National University of Singapore, Singapore, Singapore; ${ }^{2}$ University Medical Center, Utrecht, Netherlands; ${ }^{3}$ Centre for Biomedical Ethics, National University of Singapore, Singapore, Singapore

\subsection{6/spcare-2019-ACPICONGRESSABS.123}

Collusion in the healthcare setting occurs when a patient's loved ones seek healthcare professionals' cooperation in hiding or moderating the disclosure of a serious illness from the patient with capacity to make their own healthcare decisions. Collusion more commonly occurs in patients who are older, perceived as vulnerable and in need of protection from the harsh truth. When collusion occurs, the patient is excluded from the decision-making process, their autonomy is suspended, and advance care planning is not even an option. Collusion may be justifiable if the doctor exercises therapeutic privilege and withholds diagnostic and/or prognostic information from the patient because of concerns that the patient may be seriously harmed physically or psychologically, if informed. The highest court in Singapore in the recent case of Hii Chi Kok $v$ Lucien London Ooi expanded the concept of therapeutic privilege. The court endorsed the view that therapeutic privilege should not be abused by doctors to prevent patients with mental capacity from deciding for themselves just because the doctors think their choice is not in their best interests. However, it seemed to leave the door open for the possibility of triggering the therapeutic privilege if the patient is impaired in their decision-making capabilities, although still possessing mental capacity, and refuses low-risk beneficial treatment because they misunderstand the rationale treatment for it, even with appropriate assistance. Are there limits to an individual refusing beneficial treatment? Is this compatible with respecting an individual's right to make an unwise decision? Where should the line be drawn?

\section{P41 EFFECTIVENESS OF ACP TO IMPROVE PATIENT- CENTRED CARE: STUDY PROTOCOL OF A CLUSTER- RANDOMISED INTERVENTION TRIAL FOCUSSING ON NURSING HOME RESIDENTS (BEVOR-STUDY)}

${ }^{1} \mathrm{~J}$ In der Schmitten*, ${ }^{2} \mathrm{C}$ Bausewein, ${ }^{2} \mathrm{~B}$ Feddersen, ${ }^{3} \mathrm{E}$ Hummers-Pradier, ${ }^{1} \mathrm{~A}$ Icks, ${ }^{1} \mathrm{H}$ Kolbe, ${ }^{4} \mathrm{~S}$ Laag, ${ }^{5} \mathrm{G}$ Marckmann, ${ }^{6} \mathrm{G}$ Meyer, ${ }^{3} \mathrm{~F}$ Nauck, ${ }^{6} \mathrm{~J}$ Schildmann, ${ }^{7} \mathrm{~K}$ Wegscheider. ${ }^{1}$ Medical Faculty, Heinrich-Heine-University, Düsseldorf, Germany; '2LMU, Munich, Germany; ${ }^{3}$ Universitätsmedizin, Göttingen, Germany; ${ }^{4}$ Barmer Ersatzkasse, Wuppertal, Germany; ${ }^{5}$ Medical Faculty, LMU, Munich, Germany; ${ }^{6}$ Martin-Luther-Universität, Halle-Wittenberg, Germany; ${ }^{7}$ Universitätsklinikum Eppendorf, Hamburg, Germany

\subsection{6/spcare-2019-ACPICONGRESSABS.124}

Background Advance Care Planning (ACP) has been shown to increase prevalence of advance directives in German nursing homes $(\mathrm{n} / \mathrm{h})$ and therefore been introduced by recent legislation for $\mathrm{n} / \mathrm{h}$ residents covered by sickness funds. However, clinical benefits and costs of ACP for $n / h$ residents have not been studied in German speaking countries yet.

Methods cRCT in 4 study centres, each comprising $11 \mathrm{n} / \mathrm{h}$ $(3.520 \mathrm{n} / \mathrm{h}$ residents altogether). $22 \mathrm{n} / \mathrm{h}$ will be randomised to the ACP intervention, the other half continue with usual care. The complex ACP intervention consists of comprehensive 